\title{
Forced oscillation of nonlinear fractional differential equations with damping term
}

\author{
Jichen Yang, Anping Liu and Ting Liu*
}

${ }^{\text {*Correspondence: }}$

liuting4148@vip.sina.com

School of Mathematics and Physics,

China University of Geosciences,

Lumo Road, Wuhan, 430074, China

\begin{abstract}
In this article, we study forced oscillatory properties of solutions to nonlinear fractional differential equations with a damping term. Based on the properties of the Riemann-Liouville fractional derivative, we establish a sufficient condition for oscillation of all solutions.
\end{abstract}

MSC: 34A08; 34C10; 34K11

Keywords: forced oscillation; fractional differential equations; damping

\section{Introduction}

The subject of fractional calculus (that is, calculus of integrals and derivatives of any arbitrary real or complex order) has gained considerable popularity and importance during the past three decades or so, due mainly to its demonstrated applications in numerous seemingly diverse and widespread fields of science and engineering. It does indeed provide several potentially useful tools for solving differential and integral equations, and various other problems involving special functions of mathematical physics as well as their extensions and generalizations in one and more variables.

The concept of fractional calculus is popularly believed to have stemmed from a question raised in the year 1695 by Marquis de L?Hôpital (1661-1704) to Gottfried Wilhelm Leibniz (1646-1716), which sought the meaning of Leibniz?s (currently popular) notation $\frac{d^{n} y}{d x^{n}}$ for the derivative of order $n \in \mathrm{N}_{0}:=\{0,1,2, \ldots\}$ when $n=\frac{1}{2}$ (What if $n=\frac{1}{2}$ ?). In his reply, dated 30 September 1695, Leibniz wrote to L?Hôpital as follows: ?... This is an apparent paradox from which, one day, useful consequences will be drawn....?

In addition, of course, to the theories of differential, integral, and integro-differential equations, and special functions of mathematical physics as well as their extensions and generalizations in one and more variables, some of the areas of present day applications of fractional calculus include fluid flow, rheology, dynamical processes in self-similar and porous structures, diffusive transport akin to diffusion, electrical networks, probability and statistics, control theory of dynamical systems, viscoelasticity, electrochemistry of corrosion, chemical physics, optics and signal processing, and so on.

Recently, there have been some books on the subject of fractional calculus and fractional differential equations, such as $[1,2]$. Many papers have investigated some aspects of fractional differential equations, such as the existence and uniqueness of solutions to Cauchy type problems, the methods for explicit and numerical solutions, and the stability of solutions.

( 2015 Yang et al; licensee Springer. This is an Open Access article distributed under the terms of the Creative Commons Attribution License (http://creativecommons.org/licenses/by/4.0), which permits unrestricted use, distribution, and reproduction in any medium, provided the original work is properly credited. 
However, to the best of our knowledge very little is known regarding the forced oscillatory behavior of fractional differential equations up to now. On forced oscillation theory of fractional differential equations only a few of papers have been published, such as [3-6]. We establish a sufficient condition for forced oscillation of all solutions by using the properties of the Riemann-Liouville fractional derivative. As far as we know, this approach has never been used in other papers.

In this paper, we study forced oscillatory properties of solutions to nonlinear fractional differential equations with damping,

$$
\left(D_{0+}^{1+\alpha} y\right)(t)+p(t)\left(D_{0+}^{\alpha} y\right)(t)+q(t) f(y(t))=g(t), \quad t>0,
$$

with initial condition $\left(I_{0+}^{1-\alpha} y\right)(0+)=b, b$ is a real number, where $\alpha \in(0,1)$ is a constant, $D_{0+}^{\alpha} y$ is the Riemann-Liouville fractional derivative of order $\alpha$ of $y$.

We will use the following conditions:

(A) $p(t) \in C\left(\mathrm{R}^{+}, \mathrm{R}\right), q(t) \in C\left(\mathrm{R}^{+}, \mathrm{R}^{+}\right), f \in C(\mathrm{R}, \mathrm{R})$, and $f(u) / u>0$ for all $u \neq 0$, $g(t) \in C\left(\mathrm{R}^{+}, \mathrm{R}\right)$.

Definition 1.1 The solution $y$ of problem (1.1) is called oscillatory if it is neither eventually positive nor eventually negative. Otherwise, it is called non-oscillatory.

\section{Preliminaries and lemmas}

In this section, we introduce the definitions of fractional integral and fractional derivative. There are several kinds of definitions of fractional integrals and fractional derivatives [1]. In this article, we use Riemann-Liouville definition.

Definition 2.1 The Riemann-Liouville fractional integral $I_{a+}^{\alpha} y$ of order $\alpha \in \mathrm{R}^{+}$is defined by

$$
\left(I_{a+}^{\alpha} y\right)(t)=\frac{1}{\Gamma(\alpha)} \int_{a}^{t}(t-v)^{\alpha-1} y(v) d v, \quad t>a, \alpha \in \mathrm{R}^{+} .
$$

Here $\Gamma(\alpha)$ is the gamma function defined by $\Gamma(\alpha)=\int_{0}^{+\infty} s^{\alpha-1} e^{-s} d s$ for $\alpha>0, a \in \mathrm{R}$. This integral is called the left-sided fractional integral.

Definition 2.2 The Riemann-Liouville fractional derivative $D_{a+}^{\alpha} y$ of order $\alpha \in \mathrm{R}^{+}$is defined by

$$
\left(D_{a+}^{\alpha} y\right)(t)=\frac{1}{\Gamma(n-\alpha)} \frac{d^{n}}{d t^{n}} \int_{a}^{t}(t-v)^{-\alpha+n-1} y(v) d v, \quad t>a, \alpha \in \mathrm{R}^{+}
$$

with $n=[\alpha]+1$, where $[\alpha]$ means the integer part of $\alpha$.

Lemma $2.3([1])$ Let $\alpha \in(0,1)$ and $\left(I_{a+}^{1-\alpha} y\right)(t)$ be the fractional integral $(2.1)$ of order $1-\alpha$, then

$$
\left(I_{a+}^{\alpha} D_{a+}^{\alpha} y\right)(t)=y(t)-\frac{\left(I_{a+}^{1-\alpha} y\right)(a)}{\Gamma(\alpha)}(t-a)^{\alpha-1}
$$


Lemma 2.4 ([1]) Let $\alpha \geq 0, m \in \mathrm{N}$, and $D=d / d x$. If the fractional derivatives $\left(D_{a^{+}}^{\alpha} y\right)(x)$ and $\left(D_{a+}^{\alpha+m} y\right)(x)$ exist, then

$$
\left(D^{m} D_{a+}^{\alpha} y\right)(x)=\left(D_{a+}^{\alpha+m} y\right)(x)
$$

\section{Main results}

Theorem 3.1 Suppose that (A) and the following conditions hold:

$$
\begin{aligned}
& \liminf _{t \rightarrow+\infty} \int_{0}^{t} \frac{(t-w)^{\alpha-1}}{V(w)}\left(M+\int_{t_{0}}^{w} g(s) V(s) d s\right) d w<0, \\
& \limsup _{t \rightarrow+\infty} \int_{0}^{t} \frac{(t-w)^{\alpha-1}}{V(w)}\left(M+\int_{t_{0}}^{w} g(s) V(s) d s\right) d w>0,
\end{aligned}
$$

here $V(s)=\exp \int_{t_{0}}^{s} p(v) d v, M$ is an arbitrary constant. Then each solution of problem (1.1) oscillates.

Proof For the sake of contradiction, let $y(t)$ be a non-oscillatory solution of (1.1). Without loss of generality, we can assume that there exists $T>0, t_{0} \geq T$, such that $y(t)>0$ for all $t \geq t_{0}$. According to (1.1) and (A), the following inequality is satisfied:

$$
\begin{aligned}
{\left[\left(D_{0+}^{\alpha} y\right)(t) V(t)\right]^{\prime} } & =\left(D_{0+}^{1+\alpha} y\right)(t) V(t)+\left(D_{0+}^{\alpha} y\right)(t) p(t) V(t) \\
& =-q(t) f(y(t)) V(t)+g(t) V(t) \\
& <g(t) V(t) .
\end{aligned}
$$

Integrating both sides of the above inequality from $t_{0}$ to $t$, we get

$$
\left(D_{0+}^{\alpha} y\right)(t) V(t)<\left(D_{0+}^{\alpha} y\right)\left(t_{0}\right) V\left(t_{0}\right)+\int_{t_{0}}^{t} g(s) V(s) d s=M+\int_{t_{0}}^{t} g(s) V(s) d s,
$$

where $M=\left(D_{0+}^{\alpha} y\right)\left(t_{0}\right) V\left(t_{0}\right)$. From Lemma 2.3 and (3.3), we can obtain

$$
\begin{aligned}
y(t) & <\frac{\left(I_{0+}^{1-\alpha} y\right)(0)}{\Gamma(\alpha)} t^{\alpha-1}+I_{0+}^{\alpha}\left[\frac{M}{V(t)}+\frac{1}{V(t)} \int_{t_{0}}^{t} g(s) V(s) d s\right] \\
& =\frac{b}{\Gamma(\alpha)} t^{\alpha-1}+\frac{1}{\Gamma(\alpha)} \int_{0}^{t} \frac{(t-w)^{\alpha-1}}{V(w)}\left(M+\int_{t_{0}}^{w} g(s) V(s) d s\right) d w .
\end{aligned}
$$

Taking $t \rightarrow+\infty$, from the above inequality, we can obtain

$$
\begin{aligned}
\liminf _{t \rightarrow+\infty} y(t) \leq & \liminf _{t \rightarrow+\infty} \frac{b}{\Gamma(\alpha)} t^{\alpha-1} \\
& +\liminf _{t \rightarrow+\infty} \frac{1}{\Gamma(\alpha)} \int_{0}^{t} \frac{(t-w)^{\alpha-1}}{V(w)}\left(M+\int_{t_{0}}^{w} g(s) V(s) d s\right) d w \\
< & 0,
\end{aligned}
$$

which contradicts the assumption that $y(t)>0$. 
On the other hand, we can assume that there exists $T>0, t_{0} \geq T$, such that $y(t)<0$ for all $t \geq t_{0}$. According to (1.1) and (A), the following inequality is satisfied:

$$
\begin{aligned}
{\left[\left(D_{0+}^{\alpha} y\right)(t) V(t)\right]^{\prime} } & =\left(D_{0+}^{1+\alpha} y\right)(t) V(t)+\left(D_{0+}^{\alpha} y\right)(t) p(t) V(t) \\
& =-q(t) f(y(t)) V(t)+g(t) V(t) \\
& >g(t) V(t)
\end{aligned}
$$

Integrating both sides of the above inequality from $t_{0}$ to $t$, we get

$$
\left(D_{0+}^{\alpha} y\right)(t) V(t)>\left(D_{0+}^{\alpha} y\right)\left(t_{0}\right) V\left(t_{0}\right)+\int_{t_{0}}^{t} g(s) V(s) d s=M+\int_{t_{0}}^{t} g(s) V(s) d s,
$$

where $M=\left(D_{0+}^{\alpha} y\right)\left(t_{0}\right) V\left(t_{0}\right)$. From Lemma 2.3 and (3.4), we can obtain

$$
\begin{aligned}
y(t) & >\frac{\left(I_{0+}^{1-\alpha} y\right)(0)}{\Gamma(\alpha)} t^{\alpha-1}+I_{0+}^{\alpha}\left[\frac{M}{V(t)}+\frac{1}{V(t)} \int_{t_{0}}^{t} g(s) V(s) d s\right] \\
& =\frac{b}{\Gamma(\alpha)} t^{\alpha-1}+\frac{1}{\Gamma(\alpha)} \int_{0}^{t} \frac{(t-w)^{\alpha-1}}{V(w)}\left(M+\int_{t_{0}}^{w} g(s) V(s) d s\right) d w .
\end{aligned}
$$

Taking $t \rightarrow+\infty$, from the above inequality, we can obtain

$$
\begin{aligned}
\limsup _{t \rightarrow+\infty} y(t) \geq & \limsup _{t \rightarrow+\infty} \frac{b}{\Gamma(\alpha)} t^{\alpha-1} \\
& +\limsup _{t \rightarrow+\infty} \frac{1}{\Gamma(\alpha)} \int_{0}^{t} \frac{(t-w)^{\alpha-1}}{V(w)}\left(M+\int_{t_{0}}^{w} g(s) V(s) d s\right) d w \\
> & 0,
\end{aligned}
$$

which contradicts the assumption that $y(t)<0$. This proof is complete.

\section{Example}

Example 4.1 Consider the fractional differential equation

$$
\left(D_{0+}^{\frac{3}{2}} y\right)(t)-\left(D_{0+}^{\frac{1}{2}} y\right)(t)+t^{2} y e^{y}=e^{2 t} \sin t, \quad t>0,
$$

where $\alpha=\frac{1}{2}, p(t)=-1, q(t)=t^{2}, f(u)=u e^{u}, V(s)=e^{t_{0}-s}, g(t)=e^{2 t} \sin t$. Then

$$
\int_{t_{0}}^{w} g(s) V(s) d s=\int_{t_{0}}^{w} e^{2 s} \sin s \cdot e^{t_{0}-s} d s=\int_{t_{0}}^{w} e^{t_{0}+s} \sin s d s .
$$

Set $t_{0}=\frac{\pi}{4}$. Hence, we can obtain

$$
\begin{aligned}
& \int_{0}^{t} \frac{(t-w)^{\alpha-1}}{V(w)}\left(M+\int_{t_{0}}^{w} g(s) V(s) d s\right) d w \\
& =\int_{0}^{t}(t-w)^{-\frac{1}{2}} e^{w-\frac{\pi}{4}}\left(M+\int_{\frac{\pi}{4}}^{w} e^{\frac{\pi}{4}+s} \sin s d s\right) d w \\
& =\int_{0}^{t}(t-w)^{-\frac{1}{2}}\left(M e^{w-\frac{\pi}{4}}+\frac{\sqrt{2}}{2} e^{2 w} \sin \left(w+\frac{\pi}{4}\right)\right) d w
\end{aligned}
$$


Set $t-w=s^{2}$, then the above integral can be written as the following form:

$$
\begin{aligned}
\int_{\sqrt{t}}^{0} \frac{1}{s}\left(M e^{t-s^{2}-\frac{\pi}{4}}+\frac{\sqrt{2}}{2} e^{2\left(t-s^{2}\right)} \sin \left(t-s^{2}+\frac{\pi}{4}\right)\right)(-2 s) d s \\
=2 M e^{t-\frac{\pi}{4}} \int_{0}^{\sqrt{t}} e^{-s^{2}} d s+\sqrt{2} e^{2 t} \int_{0}^{\sqrt{t}} e^{-2 s^{2}} \sin \left(t-s^{2}+\frac{\pi}{4}\right) d s \\
=2 M e^{t-\frac{\pi}{4}} \int_{0}^{\sqrt{t}} e^{-s^{2}} d s+\sqrt{2} e^{2 t} \sin \left(t+\frac{\pi}{4}\right) \int_{0}^{\sqrt{t}} e^{-2 s^{2}} \cos s^{2} d s \\
\quad-\sqrt{2} e^{2 t} \cos \left(t+\frac{\pi}{4}\right) \int_{0}^{\sqrt{t}} e^{-2 s^{2}} \sin s^{2} d s
\end{aligned}
$$

Let $t \rightarrow+\infty$, because $\left|e^{-2 s^{2}} \cos s^{2}\right| \leq e^{-2 s^{2}}$ and $\lim _{t \rightarrow+\infty} \int_{0}^{\sqrt{t}} e^{-2 s^{2}} d s=\frac{\sqrt{2 \pi}}{4}$, we know that $\lim _{t \rightarrow+\infty} \int_{0}^{\sqrt{t}} e^{-2 s^{2}} \cos s^{2} d s$ is convergent. Similarly, $\lim _{t \rightarrow+\infty} \int_{0}^{\sqrt{t}} e^{-2 s^{2}} \sin s^{2} d s$ is convergent as well. Set $\lim _{t \rightarrow+\infty} \int_{0}^{\sqrt{t}} e^{-2 s^{2}} \cos s^{2} d s=A, \lim _{t \rightarrow+\infty} \int_{0}^{\sqrt{t}} e^{-2 s^{2}} \sin s^{2} d s=B$.

Select the sequence $\left\{t_{k}\right\}=\left\{\frac{3 \pi}{2}-\frac{\pi}{4}+2 k \pi-\arctan \frac{-B}{A}\right\}, \lim _{k \rightarrow \infty} t_{k}=+\infty$, then we calculate

$$
\begin{aligned}
& \lim _{k \rightarrow \infty}\left\{e ^ { t _ { k } } \left[2 M e^{-\frac{\pi}{4}} \int_{0}^{\sqrt{t_{k}}} e^{-s^{2}} d s+\sqrt{2} e^{t_{k}}\left(\sin \left(t_{k}+\frac{\pi}{4}\right) \int_{0}^{\sqrt{t_{k}}} e^{-2 s^{2}} \cos s^{2} d s\right.\right.\right. \\
& \left.\left.\left.-\cos \left(t_{k}+\frac{\pi}{4}\right) \int_{0}^{\sqrt{t_{k}}} e^{-2 s^{2}} \sin s^{2} d s\right)\right]\right\} .
\end{aligned}
$$

Firstly, we consider the following limit:

$$
\begin{aligned}
& \lim _{k \rightarrow \infty}\left\{\sin \left(t_{k}+\frac{\pi}{4}\right) \int_{0}^{\sqrt{t_{k}}} e^{-2 s^{2}} \cos s^{2} d s-\cos \left(t_{k}+\frac{\pi}{4}\right) \int_{0}^{\sqrt{t_{k}}} e^{-2 s^{2}} \sin s^{2} d s\right\} \\
& =A \cdot \lim _{k \rightarrow \infty} \sin \left(\frac{3 \pi}{2}+2 k \pi-\arctan \frac{-B}{A}\right)-B \cdot \lim _{k \rightarrow \infty} \cos \left(\frac{3 \pi}{2}+2 k \pi-\arctan \frac{-B}{A}\right) \\
& =A \cdot \sin \left(\frac{3 \pi}{2}-\arctan \frac{-B}{A}\right)-B \cdot \cos \left(\frac{3 \pi}{2}-\arctan \frac{-B}{A}\right) \\
& =\sqrt{A^{2}+B^{2}} \sin \left(\frac{3 \pi}{2}-\arctan \frac{-B}{A}+\arctan \frac{-B}{A}\right) \\
& =\sqrt{A^{2}+B^{2}} \sin \left(\frac{3 \pi}{2}\right) \\
& =-\sqrt{A^{2}+B^{2}} .
\end{aligned}
$$

Secondly, we know that $\lim _{k \rightarrow \infty} e^{t_{k}}=+\infty$ and $\lim _{k \rightarrow \infty} 2 M e^{-\frac{\pi}{4}} \int_{0}^{\sqrt{t_{k}}} e^{-s^{2}} d s=2 M e^{-\frac{\pi}{4}} \cdot \frac{\sqrt{\pi}}{2}=$ $\sqrt{\pi} M e^{-\frac{\pi}{4}}$. Hence, for (4.2), we have

$$
\begin{aligned}
\lim _{k \rightarrow \infty} & \left\{e ^ { t _ { k } } \left[2 M e^{-\frac{\pi}{4}} \int_{0}^{\sqrt{t_{k}}} e^{-s^{2}} d s+\sqrt{2} e^{t_{k}}\left(\sin \left(t_{k}+\frac{\pi}{4}\right) \int_{0}^{\sqrt{t_{k}}} e^{-2 s^{2}} \cos s^{2} d s\right.\right.\right. \\
& \left.\left.\left.-\cos \left(t_{k}+\frac{\pi}{4}\right) \int_{0}^{\sqrt{t_{k}}} e^{-2 s^{2}} \sin s^{2} d s\right)\right]\right\} \\
= & (+\infty) \cdot\left[\sqrt{\pi} M e^{-\frac{\pi}{4}}+(+\infty) \cdot\left(-\sqrt{A^{2}+B^{2}}\right)\right] \\
= & -\infty
\end{aligned}
$$


Then we obtain

$$
\begin{aligned}
\liminf _{t \rightarrow+\infty} & \int_{0}^{t} \frac{(t-w)^{\alpha-1}}{V(w)}\left(M+\int_{t_{0}}^{w} g(s) V(s) d s\right) d w \\
\leq & \lim _{k \rightarrow \infty}\left\{2 M e^{t_{k}-\frac{\pi}{4}} \int_{0}^{\sqrt{t_{k}}} e^{-s^{2}} d s+\sqrt{2} e^{2 t_{k}} \sin \left(t_{k}+\frac{\pi}{4}\right) \int_{0}^{\sqrt{t_{k}}} e^{-2 s^{2}} \cos s^{2} d s\right. \\
& \left.-\sqrt{2} e^{2 t_{k}} \cos \left(t_{k}+\frac{\pi}{4}\right) \int_{0}^{\sqrt{t_{k}}} e^{-2 s^{2}} \sin s^{2} d s\right\} \\
= & -\infty<0 .
\end{aligned}
$$

Similarly, selecting the sequence $\left\{t_{j}\right\}=\left\{\frac{\pi}{2}-\frac{\pi}{4}+2 j \pi-\arctan \frac{-B}{A}\right\}$, we can obtain

$$
\limsup _{t \rightarrow+\infty} \int_{0}^{t} \frac{(t-w)^{\alpha-1}}{V(w)}\left(M+\int_{t_{0}}^{w} g(s) V(s) d s\right) d w \geq+\infty>0
$$

Therefore, by Theorem 3.1 all solutions of (4.1) are oscillatory.

\section{Remark}

In this paper, we did not mention oscillation of fractional differential equation with time delay. Actually, we have considered the following equation:

$$
\left(D_{0+}^{1+\alpha} y\right)(t)+p(t)\left(D_{0+}^{\alpha} y\right)(t)+q(t) f(y(t-\tau))=g(t), \quad t>0,
$$

where $\tau \geq 0$ and (A) are satisfied. The conclusion is that if (3.1) and (3.2) hold, then each solution of problem (5.1) oscillates. That means the time delay $\tau$ in $f(y(t-\tau))$ has no effect on the oscillatory property.

However, we have not considered the problem in which time delays are on $\left(D_{0+}^{1+\alpha} y\right)(t)$ and $\left(D_{0+}^{\alpha} y\right)(t)$, since it is more complicated than discussions in this paper. In our future research, we would like to discuss this case and hope to acquire the desired results.

\section{Competing interests}

The authors declare that they have no competing interests.

\section{Authors? contributions}

The first author discovered the topic and the main ideas for the proof of the paper and did the actual writing. All authors discussed the paper together. The second and the third authors discovered some helpful ideas for the proof of this paper and checked the proof of the paper. All authors read and approved the final manuscript.

\section{Acknowledgements}

This research was partially supported by grants from the National Basic Research Program of China, No. 2011CB710604 and by the Science Foundation for The Excellent Youth Scholars of Ministry of Education of China, No. 007-G1323521462.

Received: 10 June 2014 Accepted: 19 December 2014 Published online: 13 January 2015

\section{References}

1. Kilbas, AA, Srivastava, HM, Trujillo, JJ: Theory and Applications of Fractional Differential Equations. Elsevier, Amsterdam (2006)

2. Samko, SG, Kilbas, AA, Marichev, OI: Fractional Integrals and Derivatives: Theory and Applications. Elsevier, Amsterdam (1993)

3. Chen, DX, Qu, PX, Lan, YH: Forced oscillation of certain fractional differential equations. Adv. Differ. Equ. 2013, 125 (2013)

4. Feng, QH, Meng, FW: Oscillation of solutions to nonlinear forced fractional differential equations. Electron. J. Differ. Equ. 2013, 169 (2013)

5. Grace, SR, Agarwal, RP, Wong, PJY, Zafer, A: On the oscillation of fractional differential equations. Fract. Calc. Appl. Anal. $15,222-231(2012)$ 
6. Han, ZL, Zhao, YG, Sun, Y, Zhang, C: Oscillation for a class of fractional differential equation. Discrete Dyn. Nat. Soc. 2013, Article ID 390282 (2013)

Submit your manuscript to a SpringerOpen ${ }^{\circ}$ journal and benefit from:

- Convenient online submission

- Rigorous peer review

- Immediate publication on acceptance

- Open access: articles freely available online

- High visibility within the field

- Retaining the copyright to your article 